\title{
Retention Force of Complete Palate Coverage and Palate-less Dentures in vitro
}

\author{
Fumio TERAOKA, Masafumi NAKAGAWA and Junzo TAKAHASHI \\ Division of Oromaxillofacial Regeneration, Course for Integrated Oral Sciences and Stomatology, Osaka University Gradu- \\ ate School of Dentistry, 1-8 Yamadaoka, Suita, Osaka, 565-0871 Japan \\ Corresponding author, E-mail:terachan@dent.osaka-u.ac.jp
}

Received October 14, 2003/Accepted December 26, 2003

\begin{abstract}
The purpose of this study was to investigate the retention force of complete palate coverage and palate-less dentures made with three polymerization systems (DS system, SR-Ivocap system, microwave polymerization system) in vitro. The retention force between each denture and a polyurethane model by intervening artificial saliva was measured using a tensile tester. In addition, discrepancies between the denture base and the stone cast were measured at several points. The retention force of complete palate coverage and palate-less dentures made with the 3 polymerization systems shows that the DS system had a greater retention force than the SR-Ivocap system and the microwave system. Moreover, the retention force of palate-less dentures was greater than that of complete dentures only in the case of the DS system. The interaction of the polymerization system and the denture type also had a statistically significant effect on the retention force. Dentures made with the DS system had smaller discrepancies compared with dentures processed with the other systems. The retention force correlated very closely with discrepancies at the denture border and the residual ridge.
\end{abstract}

Key words: Retention force, Complete palate coverage denture, Palate-less denture

\section{INTRODUCTION}

When designing a maxillary denture, complete palatal coverage has been generally extended to near the vibration line. However, this denture design is not always advantageous to denture wearers when these dentures induce gag reflexes and interfere with the perception of heat and taste. The influence of saliva viscosity, palatal form, denture design and texture of the fitting surface has been demonstrated ${ }^{1-4)}$. Shannon et $a l^{5)}$ reported that usual coverage of palatal tissues by a maxillary complete denture exerts a significant depressant affect on parotid salivary flow as elicited with candy drops. It was also reported that a reduction in the palatal coverage positively influenced a patient's sense of taste and an occasional gagging tendency disappeared ${ }^{6-8)}$. Consequently, chewing ability, taste perception, swallowing and phonetics are significant factors that contribute to the quality of life for denture wearers. Moreover, adequate retention of maxillary dentures is important clinically.

Oral candidiasis frequently occurs in individuals with dry mouth syndrome (xerostomia) and in denture wearers ${ }^{9)}$. It was also reported that a denture wearer's susceptibility to oropharyngeal candidiasis may be due to enhanced adherence of Candida species to acrylic, reduced saliva flow under the surfaces of denture fittings, improperly fitted dentures, or poor oral hygiene ${ }^{10-13)}$. Moreover, Epstein et al. ${ }^{14)}$ reported that the complications of xerostomia arise due to the effect of oral secretions on microbial colonization of the oral cavity, reduction in denture retention, mucosal dehydration and atrophy, reduced clearance rates of substances from the mouth, and reduction lubrication of oral tissues. Thus the palate-less dentures will be more advantageous than conventional complete palate coverage dentures in clinical use.

The purpose of this study was to investigate the retention force of complete palate coverage and palate-less dentures made with three polymerization systems in vitro.

\section{MATERIALS AND METHODS}

\section{Trial denture fabrication}

Sixty edentulous maxillary stone casts were prepared by pouring mixtures of dental stone (Plaston L, GC Corp., Tokyo, Japan) into a negative rubber mold (H3-402U, Nissin Dental Products Inc., Kyoto, Japan). By following conventional dental procedures, plastic artificial teeth (Endure HS S30, Shofu Corp., Kyoto, Japan) were set in wax (Paraffin wax, GC Corp, Tokyo, Japan) on the stone cast to make the maxillary trial complete palate coverage and palate-less dentures. The area of palatal reduction for the palate-less denture was $7.0 \mathrm{~cm}^{2}$ (Fig. 1). A silicone impression material (TSE 3455T, Toshiba Silicone Corp., Tokyo, Japan) was used to make two negative molds of each trial denture ${ }^{15)}$.

The original trial denture and the cast were removed from the silicone molds. A new set of artificial teeth and a new cast were placed in each mold. The cast and mold were held together while molten wax was poured through a hole in the mold. After 
the wax hardened, the mold was removed leaving a new trial denture on the cast. Thirty nearly identical complete palate coverage and palate-less trial dentures were made in this manner. 'The trial dentures were randomly divided into 3 groups (DS system, SR-Ivocap system, and microwave system) of $10 \mathrm{com}$ plete palate coverage and palate-less dentures. Each group was processed by 1 of the 3 systems and each system used its own unique formulation of acrylic resin. The DS system and the microwave polymerization method are our original polymerization systems ${ }^{16-18)}$.

\section{Denture fabrication}

DS system ${ }^{18)}$

The 20 trial dentures fabricated by this system (Okamoto Dental Corp., Shiga, Japan) were made into resin dentures by following the manufacturer's directions. The system has the following five properties at the same time: (1) a reduced amount of free

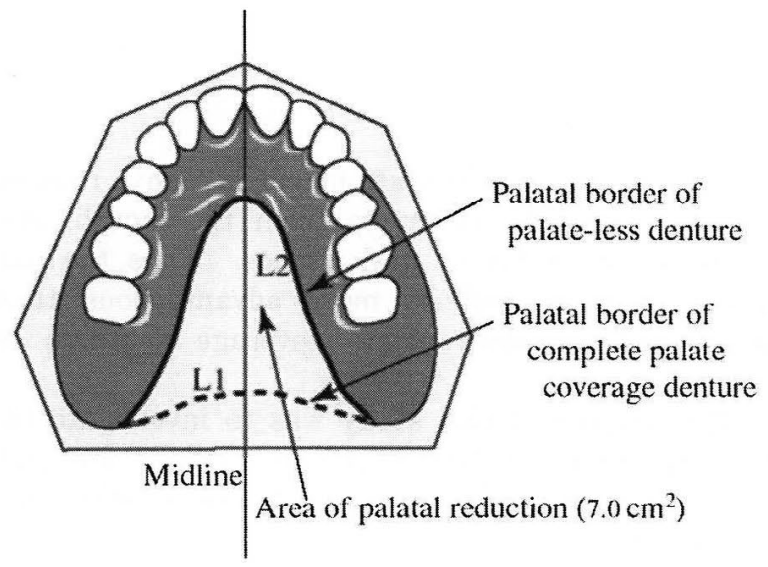

Fig. 1 Schematic diagram of a palate-less denture. Length of palatal border of complete (L1; brokenline) and palate-less (L2; solid line) dentures. water in the stone cast and gypsum mold; (2) the resin dough $(\mathrm{P} / \mathrm{L}=28 \mathrm{~g} / 10 \mathrm{~g})$ (DS resin, Okamoto Dental Corp., Shiga, Japan) must be injected and held under a pressure of about $3.5 \mathrm{MPa}$ until the polymerization is complete; (3) uses the minimum temperature necessary to completely polymerize the monomer; (4) initiate polymerization evenly and uniformly from the surface of the cast upward so the resin in the sprue is the last to harden; and (5) immediately initiate polymerization after the resin dough is injected into the mold space.

\section{SR-Ivocap system ${ }^{19-21}$}

The 20 trial dentures made by this system were made into resin dentures by following the manufacturer's introductions precisely. A capsule that contained the recommended Ivocap resin (Ivoclar North America, Inc., Amherst, N.Y., USA) was used and the ratio was $20 \mathrm{~g}$ of powder to $30 \mathrm{ml}$ of liquid.

Microwave polymerization system ${ }^{22-24)}$

Twenty trial dentures were invested in a fiberreinforced polyester (FRP) flask (GC, Corp., Tokyo, Japan). Next, conventional dental laboratory procedures were followed to invest, boil-out, coat the stone with irreversible hydrocolloid separating material, and pack the resin. The mixture of microwaveable resin (MC resin, GC Corp., Tokyo, Japan) was proportioned at 2.5 polymer: 1 monomer by weight $(\mathrm{P} / \mathrm{L}=25 \mathrm{~g} / 10 \mathrm{~g})$. After the resin was packed, the flask was placed on its base on the revolving table in a microwave oven (RE-Z2, Sharp Corp., Osaka, Japan) and irradiated for $1.5 \mathrm{~min}$ at $500 \mathrm{~W}$. The flask was then placed on its top and irradiated for another $1.5 \mathrm{~min}$. The flask was then allowed to cool to room temperature before recovering the cast and denture.

\section{Retention force measurement}

Fig. 2 shows a photograph (A) and schematic dia-
(A)

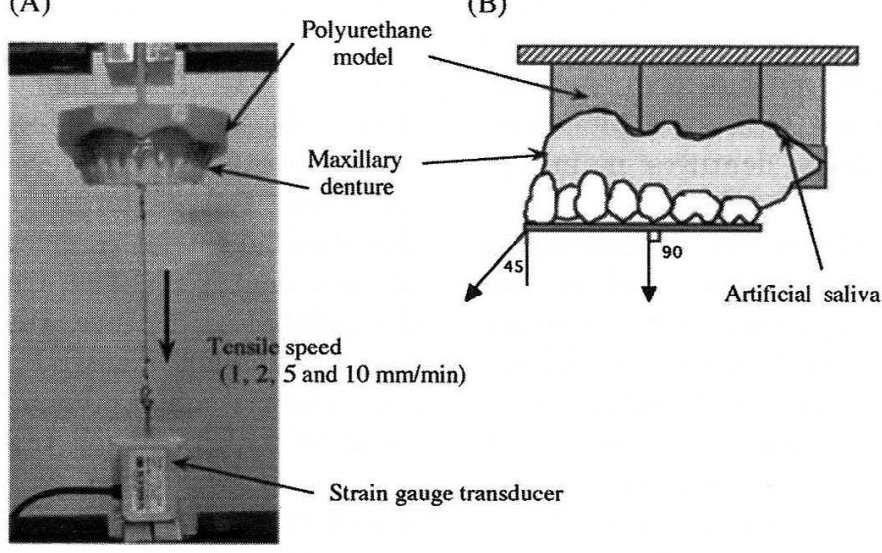

Fig. 2 Photograph (A) and schematic diagram (B) of measuring retention force. 
gram (B) of the measuring retention force. The retention force between the denture and a polyurethane model (402U, Nissin Dental Products Inc., Kyoto, Japan) by intervening with $1 \mathrm{ml}$ of artificial saliva (Salivaht, Teijin Pharma Ltd., Tokyo, Japan) was measured using a tensile tester (AGS-500D, Shimazu, Kyoto, Japan) after applying a compressive load of 5 $\mathrm{N}$ for $1 \mathrm{~min}$. Shore hardness for the polyurethane was 50. The experiments were carried out at $1,2,5$ and $10 \mathrm{~mm} / \mathrm{min}$ tensile speeds using artificial saliva of $3.89 \times 10^{-6}$ and $5.23 \times 10^{-6} \mathrm{~m}^{2} / \mathrm{sec}$ viscosity from the vertical and $45^{\circ}$ directions to the occlusal plane as shown in Fig. 2. The dentures were immersed in water at $37^{\circ} \mathrm{C}$ for 30 days until measurement. Statistical analyses of the retention force were accomplished by a two-way analysis of variance (ANOVA) and post hoc tests.

\section{Discrepancy measurement}

Each of the 30 polymerized complete palate coverage dentures was kept on its respective cast and sectioned parallel to its posterior border through the center of the mesiodistal width of the second molars (Fig. 3) after immersion in water at $37^{\circ} \mathrm{C}$ for 30 days. The machine used for the sectioning was a low-speed cutter (U-32, Luxo Corp., Tokyo, Japan). The discrepancy between the denture base and the stone cast was measured with a reading microscope (Measurescope, Nikon Corp., Tokyo, Japan). The points measured were at the right and left denture borders, the crest of the residual ridge on the right and left, and the midline of the palate (Fig. 3).

\section{RESULTS}

Increasing the tensile speed and viscosity of artificial saliva caused an increase in the retention force (Fig. 4). Subsequent experiments were carried out at a $5 \mathrm{~mm} / \mathrm{min}$ tensile speed using artificial saliva of 5.23 $\times 10^{-6} \mathrm{~m}^{2} / \mathrm{sec}$ viscosity.

The retention force of the complete palate coverage and palate-less dentures made with the 3 polymerization systems reveal that the DS system had a higher retention force than either the SR-Ivocap system or microwave system when loaded in the $90^{\circ}$ and

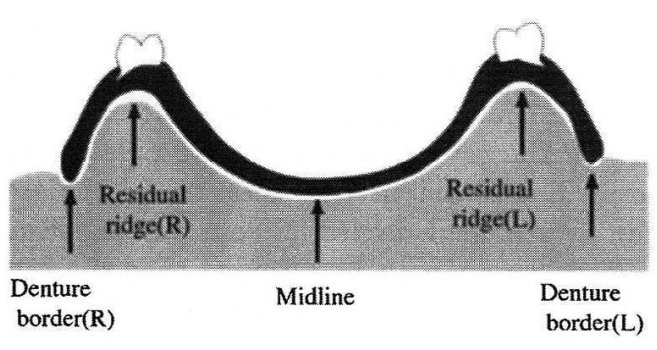

Fig. 3 Schematic diagram showing measurement locations (arrows) along the posterior palatal border cross-sectioned through second molars. $45^{\circ}$ directions (Table 1). Statistically significant differences were detected among the 3 systems when loaded in each direction $(p<0.0001)$. The means of the retention force of the two types of dentures did not differ remarkably $(p=0.4926)$. The interaction of the polymerization method and the denture type (complete palate coverage or palate-less) influenced the retention force when loaded in each direction $(\mathrm{p}<0.0001)$.

Discrepancies between the stone casts and denture bases made with the 3 polymerization systems, measured at the denture borders, residual ridges, and midline, show that the DS system had smaller discrepancies than either the SR-Ivocap system or microwave system (Fig. 5). The mean of the discrepancies for the DS system was $0.101 \mathrm{~mm}$ at the borders, $0.069 \mathrm{~mm}$ at the residual ridges, and 0.094 $\mathrm{mm}$ at the midline. Mean discrepancies at the borders, residual ridges, and midline for the lvocap system were $0.219 \mathrm{~mm}, 0.251 \mathrm{~mm}$, and $0.434 \mathrm{~mm}$ respectively; and were $0.240 \mathrm{~mm}, 0.241 \mathrm{~mm}$, and 0.195 $\mathrm{mm}$, respectively, the for the microwave system. Statistically significant differences were detected among the 3 systems at each measurement location $(\mathrm{p}<0.0001)$.

The retention forces of the complete palate coverage dentures were inversely proportional to the discrepancies at the denture border $(r=0.997)$ and residual ridge $(\mathrm{r}=0.992)$ (Fig. 6$)$.

\section{DISCUSSION}

The retention force of the complete palate coverage

Table 1 Mean values and standard deviations in $\mathrm{N}$ of retention force of dentures when loaded in 90 and $45^{\circ}$ directions (mean values designated with the same superscript are not statistically different, $\mathrm{p}>0.05)$

\begin{tabular}{c|c|c}
\hline Loading direction & Group & Mean (N) \\
\hline \multirow{5}{*}{$90^{\circ}$ direction } & DS system & \\
& Complete denture & $2.82^{\mathrm{a}} \pm 0.13$ \\
& Palate-less denture & $3.13^{\mathrm{b}} \pm 0.12$ \\
& Ivocap system & \\
& Complete denture & $1.22^{\mathrm{c}} \pm 0.20$ \\
& Palate-less denture & $1.10^{\mathrm{c}} \pm 0.18$ \\
& Microwave system & \\
& Complete denture & $1.12^{\mathrm{c}} \pm 0.18$ \\
& Palatc-less denture & $0.95^{\mathrm{c}} \pm 0.15$ \\
\hline \multirow{5}{*}{ direction } & DS system & \\
& Complete denture & $2.72^{\mathrm{a}} \pm 0.12$ \\
& Palate-less denture & $3.01^{\mathrm{b}} \pm 0.13$ \\
& Ivocap system & \\
& Complete denture & $1.19^{\mathrm{c}} \pm 0.18$ \\
& Palate-less denture & $1.01^{\mathrm{c}} \pm 0.16$ \\
& Microwave system & \\
& Complete denture & $1.01^{\mathrm{c}} \pm 0.16$ \\
& Palate-less denture & $0.90^{\mathrm{c}} \pm 0.14$ \\
\hline
\end{tabular}




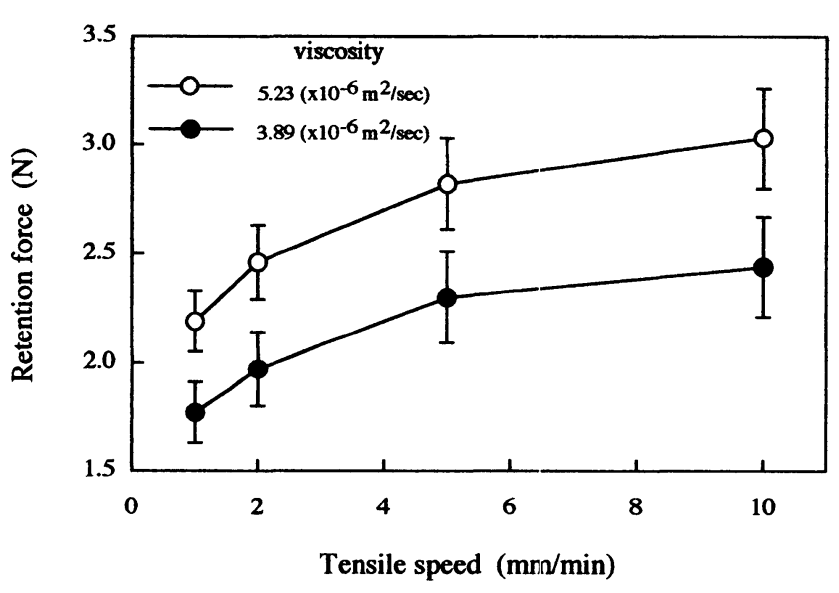

Fig. 4 Relationship between tensile speed and retention force of complete palate coverage dentures made with the DS system. (Vertical lines indicate standard deviation)

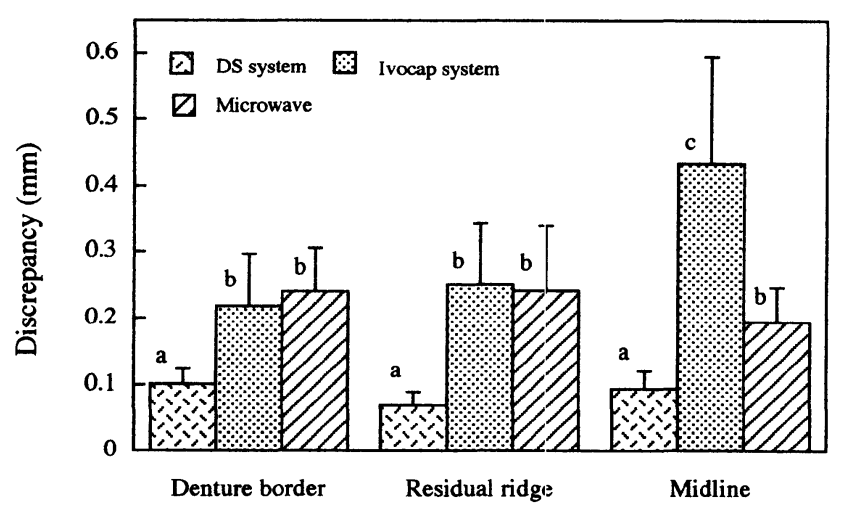

Measurement locations

Fig. 6 Relationship between the retention force and discrepancies of complete dentures made with 3 polymerization systems at each measurement location. The $r$ denotes coefficient of correlation. (Vertical lines indicate standard deviation)

and palate-less dentures made with the 3 polymerization systems showed that the DS system had a retention force of about three times greater than that of the two other systems (Table 1). In addition, the dentures fabricated with the DS system exhibited smaller discrepancies to the casts at all measurement locations than those made with the other systems (Fig. 5). These results demonstrate that the retention force of the dentures is dependent on the polymerization method. This is attributed to the differences in the mechanism of polymerization inherent in the three systems. Almost all of the conventional polymerization processes, including the SRIvocap and microwave system, are accomplished by

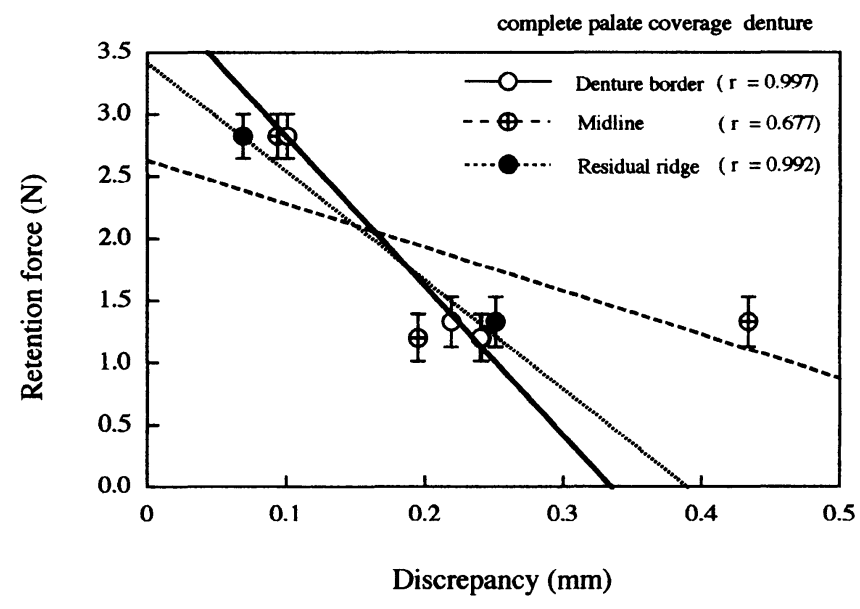

Fig. 5 Discrepancies between the stone cast and complete dentures made with the 3 polymerization systems (mean values designated with the same superscript are not statistically different, $p>0.05$ ). (Column height and vertical lines indicate mean and standard deviation, respectively)

heating the flask after the resin dough is packed or injected into the mold space. Therefore, polymerization is locally initiated in the resin dough at different times because the temperature of the mold and the resin dough is elevated by thermal conduction. Additionally, since a denture has a complicated shape with thin and thick sections, the conventional methods cannot always produce dentures that precisely fit the mucosal surface of the cast. On the other hand, the resin dough is injected into the mold space that is heated to 2 temperatures in the case of the DS system $^{18)}$. Therefore, polymerization uniformly begins from the mucosal surface of the dried stone cast which is at the higher temperature of $98^{\circ} \mathrm{C}$. Moreover, the polymerization shrinkage is largely compensated for by the resin dough being fed from the sprue that is the last to harden, so the DS system can always produce more precise dentures.

The retention force is inversely proportional to discrepancies at the denture border and residual ridge (Fig. 6). Discrepancies at the denture border correlate very closely with the retention force $(r=0.997)$. These data reveal that a sufficient retention force results from a superior denture border seal. Colòn et $a l^{2)}$ reported that the form of the palate has a direct influence on the retention of complete palate coverage dentures and will aid in the selection of the type of posterior palatal seal needed. From our experimental results, however, it may be inferred that the seal not only at the posterior palatal area but also the denture border significantly contributes to the retention of the dentures.

The retention force of palate-less dentures was greater than that of complete dentures only in the case of the DS system (Table 1). Since the seal 
around the border of the denture fabricated with the DS system is already obtained, an increase in the length of the palatal border from L1 to L2 shown in Fig. 1 will increase the retention force. Moreover, as the palate reduces, discrepancies between the stone casts and denture bases at midline tend to decrease as compared with complete palate coverage dentures. This will also result in an increase in the retention force of the palate-less dentures fabricated with the DS system. Fløystrand et $a l^{7}{ }^{7}$ reported that the ability to withstand tilting loads was insignificantly altered by a reduction in the palatal coverage during experimental dislodgement tests. Furthermore, Akeel et $a l .{ }^{25)}$ reported that palate-less dentures could be as effective as dentures with complete palatal coverage. When wearing a denture, it is necessary that a uniformly strong sealing is completely obtained in order to obtain a higher retention force. The DS system is an effective method to make palate-less dentures with a high retention force. Thus we conclude that the DS system will clinically provide an effective method of constructing palate-less dentures.

\section{REFERENCES}

1) Niedermeier WHW, Karmer R. Salivary secretion and denture retention. J Prosthet Dent 1992; 67: 211-216.

2) Colòn A, Kotwal K. Analysis of the posterior palatal seal and the palatal form as related to the retention of complete palate coverage dentures. J Prosthet Dent 1982; 47: 23-27.

3) Fløystrand F, Ørstavik JS. Retention of complete maxillary dentures as a result of changes in design. Acta Odontol Scand 1984; 42: 327-332.

4) Kikuchi M, Ghani F, Watanabe M. Method for enhancing retention on complete denture bases. J Prosthet Dent 1999; 81: 399-403.

5) Shannon IL, Terry JM, Nakamoto RY. Palatal coverage and parotid flow rate. J Prosthet Dent 1970; 24: 601-607.

6) Roy PS, Singh S. Taste threshold in complete denture wearer - A clinical study-. J Indian Dent Asso 1985; 57: $327-332$.

7) Fløystrand F, Karlson K, Saxegaard E, Ørstavik JS. Effects on retention of reducing the palatal coverage of complete maxillary dentures. Acta Odontol Scand 1986; 44: $77-83$

8) Henkin RI, Christiansen RL. Taste localization on the tongue, palate, and pharynx of normal man. J Appl Physiol 1967; 22: 316-320.

9) Cannon RD, Holmes AR, Mason, Monk BC. Oral
Candida clearance, colonization, or candidiasis. J Dent Res 1995; 74: 1152-1161.

10) Mandel ID. The function of saliva. J Dent Res 1987; 66: 623-627.

11) Sreebny LM, Valdini A. Xerostomia, a neglected symptom. Arch Intern Med 1987; 147: 1333-1337.

12) Peters NL. Snipping the thread of life. Arch Intern Med 1989; 149: 2414-2420.

13) Epstein JB, Polsky B. Oropharyngeal candidiasis, a review of its clinical spectrum and current therapies. Clin Ther 1998; 20: 40-57.

14) Epstein JB, Scully C. The role of saliva in oral health and causes and effects of xerostomia. J Can Dent Assoc 1992; 58: 217-221.

15) Teraoka F, Takahashi J. Dimensional changes of dental stones set in silicone rubber impression, Dent Mater 2000; 16: 145-149.

16) Kimura $H$, Teraoka $F$, Ohnishi $H$, Saito $T$, Yato $M$. Application of microwave for dental technique (Part 1) Dough-forming and curing of acrylic resins. J Osaka Univ Dent Sch 1983; 23: 43-50.

17) Kimura H, Teraoka F, Saito T. Application of microwave for dental technique (Part 2) Adaptability of cured acrylic resins. J Osaka Univ Dent Sch 1984; 24(1): 21-29.

18) Teraoka F, Takahashi J. Controlled polymerization system for fabricating precise dentures. J Prosthet Dent 2000; 83: 514-520.

19) Haas C. The SR Ivocap denture system. Trends Tech Contemp Dent Lab 1984; 1: 26-29.

20) Strohsver RA. Comparison of changes in vertical dimension between compression and injection molded complete dentures. J Prosthet Dent 1989; 62: 716-718.

21) Salim S, Sadamori S, Hamada T. The dimensional accuracy rectangular acrylic resin specimens cured by three denture base processing methods. J Prosthet Dent 1992; 67: 879-881.

22) Ilbay SG, Guvener S, Alkumru HN. Processing dentures using a microwave technique. J Oral Rehabil 1994; 21: 103-109.

23) Blagojevic V, Murphy VM. Microwave polymerization system for fabricating precise dentures. J Oral Rehabil 1999; 26: 804-808.

24) Keenan PL, Radford DR, Clark RK. Dimensional change in complete dentures fabricated by injection molding and microwave processing. $J$ Prosthet Dent 2003; 89: 37-44.

25) Akeel R, Assery M, AL-Dalgan S. The effectiveness of palate-less versus complete palate coverage dentures (A pilot study). Eur J Prosthodonti Restor Dent 2000; 8: 63-66. 